\title{
Partie I : Relations Suisse-Tiers Monde
}

\section{(2) OpenEdition}

1 Journals

Édition électronique

URL : http://journals.openedition.org/aspd/1051

DOI : 10.4000/aspd.1051

ISSN : 1663-9669

Éditeur

Institut de hautes études internationales et du développement

\section{Édition imprimée}

Date de publication : 1 janvier 1994

Pagination : $306-317$

ISSN : 1660-5934

\section{Référence électronique}

"Partie I : Relations Suisse-Tiers Monde », Annuaire suisse de politique de développement [En ligne],

13 | 1994, mis en ligne le 19 décembre 2012, consulté le 08 septembre 2020. URL : http://

journals.openedition.org/aspd/1051; DOI : https://doi.org/10.4000/aspd.1051

Ce document a été généré automatiquement le 8 septembre 2020.

(C) The Graduate Institute | Geneva 


\section{Partie I : Relations Suisse-Tiers Monde}

\section{Section 1 : auteurs et titres anonymes}

1 1. ABERER, Daniel. - Inwiefern widerspiegelt die schweizerische Entwicklungspolitik einen links-rechts Kompromiss ? / Referent : Jür Martin Gabriel. - St. Gallen : [s.n.], 1992. - 99 f., Mémoire de diplôme St. Gallen.

2 2.Arabisch-islamische und westliche Welt(en): Annäherungen: Referate und Diskussionen / eine Tagung der Erklärung von Bern, 4.-5. Okt, 1991 [in Zürich]. Zürich : Erklärung von Bern, 1992. - 52 p. - (Entwicklungspolitischer Beitrag ; 22).

3 3.Austausch-Projekt für Frauen : Sri Lanka, Indien, Bangladesh : dörfliche Kleinbanken und Sozialsysteme : ein Hintergrundbericht mit Reisenotizen / [Autorinnen : Frauen aus Sri Lanka, Penny Gassmann., et al.].-Zürich : EcoSolidar, 1992.-18 p.

4. BARBEY, René. - "Bibliographie = Literaturverzeichnis / avec la collab. de Viviane Maislisch", in Annuaire Suisse-Tiers Monde 1993 no. 12, p. 265-299. - Genève : Institut universitaire d'études du développement, 1993.

5 5. BAUER, Elisabeth; MOUSSA, Kathrin Karlen. - Femmes réfugiées en Suisse / publié par le Bureau fédéral de l'égalité entre femmes et hommes avec le soutien de l'office fédéral des réfugiés ODR et l'Organisation suisse d'aide aux réfugiés OSAR. - Berne : Bureau fédéral de l'égalité entre femmes et hommes, 1993. - 396 p.

6.Bekämpfung der Geldwäscherei : Modellfall Schweiz? / Hrsg. von Mark Pieth ; mit Beiträge von Hanspeter Dietzi... [et al.]. - Basel [etc.] : Helbing \& Lichtenhahn Verlag, 1992.

7 7.BERGER, Fritz; WINTERBERGER, Werner. - Photography in project work : uses and limits in photo-observation. - Bern: Swiss Development Cooperation and Humanitarian Aid, Evaluation Service, 1992. - 49 p. - (A working instrument in the séries Working instruments for planning, évaluation, monitoring and transference into action (PEMT)). 

et la mobilité des populations: nouveaux enjeux pour la recherche en sciences de l'éducation / préf. de Pierre Dasen. - Carouge: Université de Genève, Faculté de psychologie et des sciences de l'éducation, 1993. - XVI, 195, XLIII p. -(Pratiques et théorie : cahiers de la section des sciences de l'éducation ; no. 68). S. - Réfugiés et formation. - Carouge : Université de Genève, Faculté de psychologie et des sciences de l'éducation, 1993. - 184 p. - (Pratiques et théorie : cahiers de la section des sciences de l'éducation ; no. 69). 10. BICHSEL, Anne. - A review of NGO political participation at the national and international level / commissioned by the Swiss Department for Development Cooperation (SDC). - Bern : Arbeitsgemeinschaft Swissaid, Fastenopfer, Brot für alle, Helvetas, 1992. -VII, $41 \mathrm{p}$.

11 11. BISAZ, Andri. - "La coopération suisse au développement dans le Sahel : quelques éléments d'information", in Bulletin de la Société neuchâteloise de géographie 1992, no. 36, p. 23-33.

12. BLANKART, Franz. - L'Afrique : quel avenir ? : discours d'ouverture prononcé à la Conférence annuelle de la Coopération auisse au développement, à Berne le 3 septembre 1992. - Berne : Office fédéral des affaires économiques extérieures, 1992.-5 p.

13 13. BLATTNER, Nikiaus ; GENBERG, Hans ; SWOBODA, Alexandre. - La place financière suisse à l'aube du 21 e siècle = Der Finanzplatz Schweiz an der Schwelle zum 21. Jahrhundert. - Einsiedeln : Programme national de recherche 28,1993. -31 p. - (Rapport de synthèse PNR $28 ; 4)$.

14.BLUMER-FRIEDEN, Ursula; STICKELBERGER, David; ZAMBONI, Marco. Pestizidhandel: internationale Abkommen und ihre Umsetzung in der Schweiz. Zürich : Universität Zürich, 1992. - IV, 125 p., Travail de diplôme.

15. BOCCO, Riccardo ; FAEHNDRICH, Hartmut ; KILPATRICK, Hilary. - Les études sur l'Afrique du Nord et l'Asie de l'Ouest en Suisse : état des lieux et plaidoyer pour une réorganisation de la formation et de la recherche selon une approche "area-study" / avec la collab. de Hildegard Brog, Benoît Vulliet. - Berne : Conseil suisse de la science, 1993. - 172 p. - (Détection avancée en politique de la recherche ; FER 135/1993).

16 16. BOLZMAN, Claudio. - Exil, dynamique socio-culturelle et participation sociale : le cas de la migration chilienne en Suisse. - Genève : Université de Genève, 1992. -2 vol. (662 p.), Thèse sciences écon. et soc.

17 17. BOLZMAN, Claudio. - Les métamorphoses de la barque: les politiques d'asile, d'insertion et de retour de la Suisse à l'égard des exilés chiliens. - Genève : Institut d'études sociales, 1993. - 142 p. - (Nouveaux cahiers de l'I.E.S. ; no. 2), Bibliogr. p. 131142.

18 18. BRAVO-BAUMANN, Heidi. - "Auswirkungen einer Liberalisierung im Agrarsektor auf Entwicklungsländer und die Schweiz", in Annuaire Suisse-Tiers Monde 1993 no. 12, p. 173-191. - Genève : Institut universitaire d'études du développement, 1993, Résumé en français p. 261.

Annuaire suisse de politique de développement, 13 | 1994 

ein Seminar organisiert von INFRAS, Zürich und dem Ethnologischen Institut der Universität Zürich, Juni 1993 / hrsg. von Barbara Hearing und Othmar Schwank. Zürich : INFRAS, 1992, 70 p. - (Infras-Themen)

19. BRAWAND, Antoine; CANCELLIERI, Pierre-Georges; PERROULAZ, Gérard. -"Statistiques", in Annuaire Suisse-Tiers Monde 1993 no. 12, p. 301 -390. - Genève : Institut universitaire d'études du développement, 1993.

20. BRUGGER, Ernst A. - "Nachhaltige Entwicklung: die notwendige Utopie", in: Annuaire Suisse-Tiers Monde 1993 no. 12, p. 207-214. - Genève : Institut universitaire d'études du développement, 1993, Résumé en français p. 262.

21. BRUSICK, Philippe. - "La Suisse et les pratiques commerciales restrictives", in Annuaire Suisse-Tiers Monde 1993 no. 12, p. 193-206. - Genève : Institut universitaire d'études du développement, 1993, Résumé en allemand p. 261.

22. CATTANEO, Simone. - J'ai à te parler. - Genève : Caritas; Genève-Tiers Monde, 1992.-181 p.

23.Changing mentalities : vocational training and development cooperation : challenge for the developing countries : [a sourcebook of practical ideas and insights on human resource development and training in the Third World / J.J. Alarcon, R. Guardans, T. Hagen, R. Jenny, B.M. Villegas and other authors from 4 continents; foreword by Ambassador N. Imboden. - Zürich : Limmat-Stiftung, [1993].

24. CORMINBOEUF, Christian ; MAISLISCH, Viviane ; VULLIET, Benoît. - Recherche suisse et pays en voie de développement $=$ Schweizer Forschung und Entwicklungsländer $=$ Swiss research and developing countries : 1993-1994. -1 ère éd.

- Genève : Institut universitaire d'études du développement, Centre de Documentation, 1993. - XXIII, 419 p., Pré-titre : Who does what in Switzerland ? : [..].

25.COTTIER, Thomas. - Technologie-Kooperation nach Rio: rechtliche Rahmenbedingungen : Vortrag gehalten vor der Naturforschenden Gesellschaft in Bern vom 15. Februar 1993. - Bern : BAGE, 1993. -16 p.

26. DELACRETAZ, Olivier. - L'universel enraciné : remarques sur le racisme et l'antiracisme. - Lausanne : Cahiers de la renaissance vaudoise, 1993. - 96 p. -(Cahiers de la renaissance vaudoise; no 125).

27. DENT AN, Anne; BOLAY, Jean-Claude. - De l'urgence au développement: reconstructions après catastrophes naturelles en Amérique latine / avec la collab. de Alain Garnier. - Lausanne : Institut de recherche sur l'environnement construit (IREC) ; E.P.F.L., 1993. - 243 p. - (Rapport de recheche ; no. 104).

28.EGLI, Dominik. - Mikroökonomische Analyse der Schuldenkrise: wo stehen wir heute ?. - Einsiedeln: Schweiz. Nationalfonds, 1992. - 31 p. - (Nationales Forschungsprogramm 28. Diskussionspapier ; 9).

29.FIERZ, Gaby et al.-Türkei : Ferienland-Fluchtland.-Zürich : Rotpunktverlag, 1992.

- 166 p. - (Kleine Reihe Tourismus und Entwicklung ; 6).

30.Frauenforschung für die Entwicklungszusammenarbeit : Referate und Diskussion :

31. FREYVOGEL, Thierry A. - "Forschungs-Partnerschaft mit Entwicklungsländern", in Annuaire Suisse-Tiers Monde 1993 no. 12, p. 215-224. - Genève: Institut universitaire d'études du développement, 1993, Résumé en français p. 263. 

Welt kaputt / hrgs. von der Erklärung von Bern; mit Photos von Peter Frey und
Andreas Schwaiger-Lookat. - Zürich : Unionsverlag, cop. 1992. - 211 p.

$$
\text { Rouge et du Croissant-Rouge / avec la collab. de Hans-Peter Gasser., [et al.]. - Genève }
$$
[etc.] : Institut Henry-Dunant, 1993. - 690 p. + addendum.

32. GÄMPERLI, U. et al. - Sozio-ökonomische Feldforschung in der Dritten Welt : 8 Referate zu Methodik und Umfeld von laufenden Forschungsarbeiten / Zusammenstellung T. Kohler. - Bern: Universität Bern, Geographisches Institut, Abteilung für Kulturgeographie, 1991. - 78 p. - (Berichte zu Entwicklung und Umwelt ; 3), Gruppe für Entwicklung und Umwelt.

33.GERSTER, Richard; GUGLER, Alfred. - Schweizerische Entschuldungsmassnahmen : wo stehen wir heute ?. - Bern : Informationsdienst 3. Welt, 1993. - 21 p. -(i3w Dokument).

34. GONTARD, Jean-Pierre. - Pour une politique suisse d'aide au développement conforme à la loi : 11 propositions concrètes : document de travail pour la journée de réflexion du P.S.S. du 6 février 1993, Berne. - Genève: Fédération genevoise de coopération, 1993. - $11 \mathrm{p}$.

35.GREMINGER, Thomas. - "Die schweizerische Entwicklungszusammenarbeit 1991 : zwischen Euphorie und Ernüchterung", in : Die Volkswirtschaft vol. 65, no. 12, 1992, p. 48-53.

36.GRUETTER, Karin ; MAIER, Erika ; WOLF, Markus. - Gender-balance in der Entwicklungszusammenarbeit. - Zürich : NADEL, 1993, Travail de séminaire.

37.HÄBERLI, Christian. -GATT/Uruguay Runde und die Entwicklungsländer : Einführungsreferat anlässlich der Informationsveranstaltung der katholischen Hilfswerke der Schweiz zum Thema "Die GATT-Verträge : Segen oder Flucht für die Dritte Welt ?" vom 8. Juni 1993 in Luzern. - Bern : BAWI, 1993. - 33 p.

38.HALDIMANN, Urs ; DIETRICH, Stephan. - Unser täglich Fleisch : so essen wir die 40. HAUG, Hans. - Menschlichkeit für alle : die Weltbewegung des Roten Kreuzes und des Roten Halbmonds / unter Mitw. von Hans-Peter Gasser., [et al.]. - [Genf] ; Bern : Institut Henry-Dunant ; P. Haupt, 1993. - 715 p.

41.HAUG, Werner. - "Einflüsse der Migrationen auf die Bevölkerungsentwicklung der Schweiz", in : Migrationen aus der Dritten Welt. - Bern, [s.d.] - P. 123-145.

42.HISZOWICZ, Christine. - Schweizerische Bankpolitik. - 3. vollst. Überarb. Aufl. Bern [etc.] : P. Haupt, 1993. - 19, 558 p. - (Bank- und finanzwirtschaftliche Forschungen : Bd. 78).

43.HOEGGER, Rudolf. - Wasserschlange und Sonnenvogel: die andere Seite der Entwicklungshilfe. - Frauenfeld : Waldgut, 1993.

44.HOEGGER, Rudolf et al. - External évaluation of the Kilombero health research and support project. - Bern : [s.n.], 1993.

45.HURNI, H. ; HERREN, U. ; HUGUENIN, A. et al. - Denkanstösse zur Integration der Umwelt in der Schweizerischen Entwicklungszusammenarbeit. - Bern : University of Berne, Institute of Geography, Group for Development \& Environment, 1990. - 23 p. (Bereich Grundlagen und Konzepte). 

Entwicklungszusammenarbeit und humanitäre Hilfe (DEH). - Bern : Universität Bern, Geographisches Institut, Gruppe für Entwicklung und Umwelt, 1992. - 91 p. - (Bericht zu Entwicklung und Umwelt ; 4).

53.KLÄY, A. ; HUGUENIN, A. - Umweltverträglichkeit : Hilfsmittel und Arbeitsabläufe in der Projektplanung verschiedener Agenturen. - Bern : University of Berne, Institute of Geography, Group for Development \& Environm, 1990. - 45 p. - (Bereich Grundlagen und Konzepte. Berichte zu Entwicklung und Umwelt ; 1).

54.KUG, Peter; MESMER, Beatrix. - Von der Entwicklungshilfe zur Entwicklungspolitik. - Bern : [s.n.], 1993. - 685 p. - (Studien und Quellen ; Bd. 19)

55. MADELIN, Philippe. - L'or des dictatures. - Paris : Fayard, 1993. - 470 p. (Collection Enquêtes).

56. MARREL, Catherine. - "Die schweizerischen Direktinvestitionen im Ausland: Erhebungsmethode und Verlauf der schweizerischen Direktinvestitionen seit 1985", in Annuaire Suisse-Tiers Monde 1993 no. 12, p. 225-241. - Genève : Institut universitaire d'études du développement, 1993, Résumé en français p. 263.

57. Migration et formation : textes et documents accompagnant le cours de Micheline Rey / [éd.] Micheline Rey-von Allman, Dominique Motta. - 3e tirage. - Genève : Université de Genève, [Faculté de psychologie et des sciences de l'éducation], 1992.-IV, $247 \mathrm{p}$.

58.Migrationen aus der Dritten Welt: Ursachen - Wirkungen Handlungsmöglichkeiten / hrsg. von Walter Kälin und Rupert Moser. - 3. Auflage. - 
Bern [etc.] : P. Haupt, 1993. - 324 p. - (Publikation der Akademischen Kommission der Universität Bern).

61 59. MONT AVON, Rémy. - La première implantation industrielle de Nestlé en Chine : Nestlé Shuangcheng Ltd. - Vevey : Nestlé SA, cop. 1990. - 79 p.

60. MONTAVON, Rémy. - Nestlé en Inde, 1962-1992: de la fabrique de Moga 1962 à celles de Choladi 1967, de Najangud 1989 et de Samalkha 1992. - Vevey : Nestlé SA, cop. 1993.-76 p.

61.MOSER, Rupert. - "Migration und Fremdenfeindlichkeit", in : Migrationen aus der Dritten Welt. - Bern, 1993. - P. 189-203.

62.POLETTI, Mario. - Der Pilatus Schwindel : PC-7 und PC-9 im Sturzflug. - Basel : Terre des Hommes Schweiz; Arbeitsgemeinschaft für Rüstungskontrolle und ein Waffenausfuhrverbot (ARW), 1993. - $104 \mathrm{p}$.

63.Rechtshilfe und Kapitalflucht : eine Stellungnahme des Instituts für Sozialethik SEK und von Justitia et Pax. - Bern : Institut für Sozialethik des SEK, [ 1992]. -7 p.-(Texte/ ISE ; 10/92).

65.Seiltanz ohne Netz?: Kulturkonflikte im Alltag: Erfahrungen, Erwartungen, Ergebnisse $=$ Sur la corde raide, sans filet $?:$ conflits interculturels au quotidien : experiences, attentes, aboutissements / [Recherchen: Bennie Koprio]; [Red.: Ina Boesch, Rolf Probala]. -Zürich : Verein CH 701, c/o ProjektTATelier, 1992. -151 p.

66.Sozio-ökonomische Feldforschung in der Dritten Welt : 8 Referate zu Methodik und Umfeld von laufenden Forschungsarbeiten / T. Kohler, Herausgeber. - Bern : University of Berne, Institute of Geography, Group for Development \& Environment, 1991. - 78 p. (Bereich Grundlagen und Konzepte. Berichte zu Entwicklung und Umwelt ; 3).

67.SPEICH,Daniel ;MARKUS, John;GERBER,Thomas.-Zaire :dossieräl attention des représentants d'oeuvres d'entraide et des bureaux de consultation juridique / trad. F. Fliege. - Lausanne : Organisation suisse d'aide aux refugies (OSAR), 1992. -11,61 p. 68.STAEHELIN, Balthasar. - Völkerschauen im Zoologischen Garten Basel : 1879-1935. - Basel : Basler Afrika-Bibliographien, 1993. - 184 p. - (Beiträge zur Afrikakunde ; 11). 69.STAHELIN, Fritz R. - Bevölkerungswachstum und Umweltzerstörung aus der Sicht der Entwicklungszusammenarbeit: [Tagung der Schweizerischen Gesellschaft für Umweltschutz am 6. November 1992 in Rüschlikon]. - [s.l.] : [s.n.], 1992. -11 p.

70.Technology transfer for development: the prospects and limits of information technology / [an international conference held at the Gottlieb-Duttweiler-lnstitute in Rüschlikon/Zürich, Switzerland, 5-6 December 1991 in cooperation with Austrian Computer Society, Vienna., et al.] ; ed. : Günther Cyranek, S. O Bhatnagar. - New Delhi [etc.] : Tata McGraw-Hill, cop. 1992. - IX, 240 p.

72 71.WASESCHA, Luzius. - GATT - Die Bedeutung der Uruguay-Runde für die Schweiz : Referat gehalten vor der SPS-Fraktion der Bundesversammlung (16. März 1993). Bern : BAWI, 1993. $-10 \mathrm{p}$.

73 72.WASESCHA, Luzius. - GATT and Latin America : the view of Switzerland : summary of the presentation held at the AGLA/DAGLA-Meeting organized by Interphama at Interlaken (May 7, 1993). - Bern : BAWI, 1993. - 8 p.

74 73.WICKER, Hans-Rudolf. - Die Sprache extremer Gewalt : Studie zur Situation von gefolterten Flüchtlingen in der Schweiz und zur Therapie von Folterfolgen / erstellt im 
Auftrag des Schweizerischen Roten Kreuzes ; unter Mitarb. von Suna Cosar.. [et al.]. Bern : Universität, Institut für Ethnologie, 1993. -142 p. - (Arbeitsblätter/Institut für Ethnologie ; Nr. 6).

\section{Section 2 : Institutions privées auteurs}

74.Arbeitskreis Tourismus und Entwicklung, Basel.-Gebunden in Schweigen : Sex mit Kindern ist ein Verbrechen / Schweizer Kampagne gegen Kinderprostitution; [Red.: Maya Krell., et al]. - Basel: Arbeitskreis Tourismus und Entwicklung [etc.], 1993.-104 p.

75. Bureau fédéral de l'égalité entre femmes et hommes, Berne. - Les femmes victimes de persécutions et la notion de réfugié : comment interpréter le terme de "réfugié" figurant dans la Convention relative au statut des réfugiés et dans la loi sur l'asile ? / Christin Hausmann; [trad. : Francine Matthey]. - Berne : Bureau fédéral de l'égalité entre femmes et hommes (distrib : OOCFIM), 1992. - 53, IV p.

76. Bureau lausannois des immigrés, Lausanne. - Liste d'adresses des associations et groupements d'étrangers de Lausanne et environs. - Lausanne : Bureau lausannois des immigrés, 1992. - $54 \mathrm{p}$.

77. Büro für Zusammenarbeit mit Osteuropa (BZo), Bern. - Die Schweiz und Osteuropa: eine Dokumentation zum Unterstützungsprogramm des Bundes für die osteuropäischen Staaten = La Suisse et les pays d'Europe orientale : documentation sur le programme de soutien de la Confédération aux pays d'Europe orientale = Switzerland and Eastern Europe: documentation on the Swiss government's support programme for the States of Eastern Europe. - Bern : BZO, 1993.

78. Caritas, Genève. - Caritas : 50 ans : 1942-1992. - Genève : Caritas, [1992]. - 32 p.

79. Caritas, Genève. - Caritas : 50 ans de solidarité, 50 ans de bénévolat : 1943-1993.

81 - Genève : Caritas, [ 1993]. - 15 p.

80. Centre de documentation de la Communauté d'organisations suisses de développement, Lausanne. - Le café : dossier de presse. - [Lausanne]: Centre de documentation de la Communauté d'organisations suisses de développement, 1993. Non pag.

81. Centre d'information pour les professions relatives à la coopération au développement (CINFO), Bienne. - Cinfodossier "Grundlagen, Entwicklungszusammenarbeit und humanitäre Hilfe". - Biel : CINFO, 1993.

82. Communauté catholique suisse de travail pour les étrangers et leurs problèmes (SKAF), Lucerne. - Minorités en Suisse : informations et idées sur la façon de vivre ensemble. - Lucerne: Communauté catholique suisse de travail pour les étrangers et leurs problèmes, 1992. - 29 p. - (Documentation SKAF ; 1992/2).

83. Communauté de travail pour l'accueil et l'intégration des étrangers, Genève.

86

- Bienvenue à Genève : liste d'adresses utiles à l'intention des personnes étrangères venant vivre et travailler dans le canton de Genève. - Genève : Communauté de travail pour l'accueil et l'intégration des étrangers, 1992. - 20 p. 
84. Fastenopfer der Schweizer Katholiken, Luzern; Brot für alle, Bern. - Vive l'Afrique : Macht teilen, gemeinsam leben / Aktion 1993 Brot für alle, Fastenopfer. Luzern ; Bern : Fastenopfer der Schweizer Katholiken ; Brot für alle, [ 1993]. - [64] p.

85. Fédération genevoise de coopération (FGC), Genève. - Communication, information et développement sous la loupe / Le Village alternatif, Salon du livre du 5 au 9 mai '93. - Genève : Fédération genevoise de coopération, 1993. - 66, [12] p.

86. Flüchtlingsinformation, Dokumentationsstelle der Stiftung Gertrud Kurz, Bern. -Türkei : der"stille" Krieg geht weifer : eine Menschenrechtsdelegation aus der Schweiz berichtet: Dezember 1990-März 1991. - Bern: Flüchtlingsinformation, Dokumentationsstelle der Stiftung Gertrud Kurz, 1991. - 69 p.

87. Genève Tiers-Monde, Genève. - 1983-1993 : 10 ans d'action : 150 appuis à des initiatives locales : un bilan positif. - Genève : Genève Tiers-Monde, 1993. - 63 p.

88. InfoSud, Lausanne. - Afrique : la démocratie en question : (un choix d'articles issus de la presse internationale). - Lausanne : InfoSud, Agence de presse-documentation, [ 1992]. - Pag. mult.

89. InfoSud, Lausanne. - Pour un commerce équitable avec le tiers-monde. Lausanne : InfoSud, Agence de presse-documentation, [ 1992]. - Pag. mult.

90. Institut universitaire d'études du développement (IUED), Genève. - Aide suisse aux pays en développement et aux pays de l'Europe orientale 1991 / publié par l'Institut universitaire d'études du développement (IUED) ; sous mandat de la Direction de la coopération au développement et de l'aide humanitaire (DDA) -Schweizerische Hilfe für Entwicklungsländer und Oststaaten 1991 / hrsg. vom IUED; im Auftrag der Direktion für Entwicklungszusammenarbeit und humanitäre Hilfe (DEH). - Genève : Institut universitaire d'études du développement, 1992. - 175 p.

94 91.Institut universitaire d'études du développement (IUED), Genève. - Who does what in Switzerland : scientific coopération with developing countries : a preliminary survey of institutions, research projects, researchers / supervised by Christian Corminbceuf ; [ed.] Institute of Development studies (IUED), Swiss Academy of Sciences, Swiss Development Cooperation. - Geneva: Institut universitaire d'études du développement, 1992. - XXVII, 397 p.

92. Institut universitaire d'études du développement (IUED), Genève. - Vers un ailleurs prometteur.. : l'émigration, une réponse universelle à une situation de crise ? / sous la dir. de l'Equipe des Cahiers; en collab. avec le Laboratoire de démographie économique et sociale, Université de Genève. - Paris ; Genève : Presses universitaires de France ; Cahiers de N.U.E.D., 1993. - 391 p. - (Cahiers de l'Institut universitaire d'études du développement ; 22).

93. Intercooperation, Bern. - Gender analysis / Intercooperation, Secteur promotion des organisations populaires (POP). - Bern : Intercooperation, 1992. - $42 \mathrm{p}$.

94. Office central suisse d'aide aux réfugiés (OSAFt), Lausanne. - Pakistan: informations pour représentants d'oeuvres d'entraide aux auditions / recueillies par Hans Peter Roth; trad. : I. Pfeiffer et O. von Allmen. - Lausanne : Office central suisse d'aide aux réfugiés (OSAR), 1990.-51 p.

95. Office fédéral de l'environnement, des forêts et du paysage (OFEFP), Berne. Rapport présenté par la Suisse à la Conférence des Nations Unies sur l'environnement 
et le développement, Rio de Janeiro, juin 1992 / [réd., ill. : Vera Bueller] ; [trad. Pierre Grandjean, Jean-François Zurbriggen]. - Berne : OFEFP, 1992. - 90 p.

96. Organisation Schweiz-Dritte Welt, Orpund. - Honig / [OS3]. - Orpund: OS3, [ 1990].-16 p.

97. Reporters sans frontières (RSF), Lausanne. - L'intimidation : rapport sur les meurtres de journalistes et les pressions à rencontre de la presse turque. -Lausanne : Reporters sans frontières, 1993.-64p., Lacouv. porte : RSF en Turquie.

98.Schweizerische Flüchtlingshilfe (SFH), Zürich. - Urgent = Dringend = Urgente. Zürich : Schweiz. Flüchtlingshilfe, [ 1993]. - 21 p.

99.Schweizerisches Rotes Kreuz (SRK), Bern. - Gesundheitsarbeit in Bolivien : der eigene Weg indianischer Gemeinschaften. - Bern: SRK, Internationale Zusammenarbeit, 1993. - [24] p., [4] f. - (Publikationsreihe SRK International ; Nr. 2).

100.Swisscontact, Zürich. - Frauen und Entwicklung. - [S.I.J : Swisscontact, [s.d.] -39

p.

\section{Section 3 : publications gouvernementales}

\section{A. CONFEDERATION SUISSE : arrêtés, rapports, messages, ordonnances,...}

(Remarque : toutes les références sont disponibles également en allemand)

101. “Accord entre la Confédération suisse et la République du Paraguay concernant la promotion et la protection réciproque des investissements : conclu le 31 janvier 1992, entré en vigueur par échange de notes le 28 septembre 1992", in RO vol. 3, no. 47, 1992, p. 2384.

102. "Loi fédérale concernant la participation de la Suisse aux institutions de Bretton Woods du 4 octobre 1991", in RO vol. 3, no. 49, 1992, p. 2567.

103. "Arrêté fédéral concernant l'adhésion de la Suisse aux institutions de Bretton Woods du 4 octobre 1991", in RO vol. 3, no. 49, 1992, p. 2570.

104. "Statuts du Fonds monétaire international adoptés à Bretton Woods le 22 juillet 1944", in RO vol. 3, no. 49, 1992, p. 2571.

109 105. "Statuts de la Banque internationale pour la reconstruction et le développement adoptés à Bretton Woods le 22 juillet 1944", in RO vol. 3, no. 49, 1992, p. 2646.

110 106. "Statuts de l'Association internationale de développement adoptés à Washington le 26 janvier 1960", in RO vol. 3, no. 49, 1992, p. 2680.

111 107. "Statuts de la Société financière internationale adoptés à Washington le 25 mai 1955”, in RO vol. 3, no. 49, 1992, p. 2707.

112 108. "Ordonnance sur les droits de douane applicables aux marchandises dans le trafic avec Israël du 14 décembre 1992”, in RO vol. 1, no. 1,1993, p. 18.

109. “Arrêté fédéral portant approbation de l'Accord entre les pays de l'AELE et la Turquie du 19 mars 1992", in RO vol. 1, no. 1, 1993, p. 154. 
114 110. "Rapport sur la politique économique extérieure 92/1+2 et Messages concernant des ccords économiques internationaux du 20 janvier 1993”, in FF vol. 1, no. 7,1993, p. 293.

111. "Protocole de prorogation de l'accord commercial entre le gouvernement de la Confédération suisse et le gouvernement de la République de Cuba: conclu le 17 décembre 1992, entré en vigueur le 1 er janvier 1993”, in RO Vol. 1, no. 12,1993, p. 1094. 112. “Accord entre la Confédération suisse et la République du Cap-Vert concernant la promotion et la protection réciproque des investissements : conclu le 28 octobre 1991, entré en vigueur par échange de notes le 6 mai 1992", in RO Vol. 1, no. 13, 1993, p. 1253. 113. "Message concernant une convention de double imposition avec le Maroc", in FF vol. 2, no. 26, 1993, p. 821.

114. “Ordonnance instituant des mesures économiques à rencontre d'Haïti : du 30 juin 1993", in RO Vol. 2, no. 27, 1993, p. 2053.

119 115. “Arrêté fédéral portant approbation de l'Accord entre les Etats de l'AELE et Israël du 17 mars 1993”, in RO Vol. 2, no. 35, 1993, p. 2476.

16. “Accord entre les Etats de l'AELE et Israël : conclu à Genève le 17 septembre 1992, approuvé par l'Assemblée fédérale le 17 mars 1993, instrument de ratification déposé par la Suisse le 11 mai 1993, entré en vigueur pour la Suisse le 1er juillet 1993", in RO Vol. 2, no. 35,1993, p. 2477.

117. “Ordonnance instituant des mesures économiques à l'encontre d'Haïti du 30 juin 1993 : suspension du 8 septembre 1993”, in RO Vol. 3, no. 37, 1993, p. 2581.

118. “Traité d'extradition du 26 novembre 1880 entre la Suisse et la Grande-Bretagne : échange de notes des 28 janvier/9 mai 1932 concernant l'application du traité anglosuisse d'extradition à la Transjordanie", in RO vol. 3, no. 39,1993, p. 2745.

119. "Message concernant le renouvellement de la participation de la Suisse aux Accords généraux d'emprunt du Fonds monétaire international du 15 septembre 1993", in FF vol. 3, no. 40, 1993, p. 585.

120. “Ordonnance concernant l'exécution de l'Accord international sur le café de 1983 du 20 septembre 1993”, in RO vol. 3, no. 40, 1993, p. 2763

121. “Accord entre le Conseil fédéral suisse et le Gouvernement du Royaume du Maroc relatif aux transports internationaux par route: conclu le 24 octobre 1986, entré en vigueur par échange de notes le 28 septembre 1993", in RO vol. 3, no. 42,1993, p. 2870.

122. "Echange de lettres des 15-21 juin 1992 entre la Suisse et l'Algérie complétant l'accord des 15 janvier-28 mai 1991 concernant la dispense réciproque du visa envers certains ressortissants de l'autre Etat: entré en vigueur le 21 septembre 1992", in RO vol. 3, no. 43, 1993, p. 2895.

123. “Ordonnance instituant des mesures économiques à rencontre d'Haïti du 30 juin 1993 : remise en vigueur du 20 octobre 1993”, in RO Vol. 3, no. 45,1993, p. 2953.

\section{B. DIRECTION DE LA COOPERATION AU DEVELOPPEMENT ET DE L'AIDE HUMANITAIRE, DDA}

N.B. : Ne comprend pas les publications périodiques 
124. Aus unterschiedlichen Welten in eine gemeinsame Zukunft = De mondes différents à un futur commun: Forum Münchenwiler.-Bern: Direktion für Entwicklungszusammenarbeit und humanitäre Hilfe, cop. 1993. - 96 p. - (Schriften der DEH ; 4).

125. BETI, Dino. - Im Land der Pachamama : Bolivien am Scheideweg $=$ Au pays de la Pachamama: la Bolivie à la croisée des chemins = Nel paese della Pachamama: la Bolivia al crocevia / publié par le Service de l'information de la Direction de la coopération au développement et de l'aide humanitaire (DDA); texte: Dino Beti. Berne : DDA, cop. 1993. - 44 p.

126.BINKERT, Gregor. - Soziale Dimension der Entwicklung: speaking notes für Grundsatzdiskussion im Operationskomitee vom 14. Okt. 92. - Bern : DEH, 1992. -8 f.

127. COTTI, Flavio et al. - La Suisse et la Conférence de Rio sur l'environnement et le développement. - Berne : Direction de la coopération au développement et de l'aide humanitaire (DDA), cop. 1993. - 94 p. - (Cahiers de la DDA ; 3).

128.EL SAADAWI, Nawal ; HETATA, Sherif. -Good governance, human rights, cultural coopération with special référence to women in the south mediterranean région: report to the Swiss Development Cooperation. - Bern : [DDA], 1992.

129. Politique sectorielle de la promotion de l'artisanat et de l'industrie. - Berne : DDA, 1992. - 18 p. - (Politiques sectorielles DDA).

130. Programme transversal: développement équilibré hommes-femmes (Gender + Development) / DEH, Fachdienst Ressources humaines. - Bern : DDA, 1993.

131. Richtlinien für die Ausarbeitung und Genehmigung von Landes- und Jahresprogrammen sowie von Sektorpolitiken und -Programmen der bilateralen operationnellen EZA [Entwurf]. - Bern : DEH, 1993. - (Richtlinien DEH).

132.Sommet planète Terre (1992; Rio de Janeiro) : Konferenz der Vereinten Nationen über Umwelt und Entwicklung in Rio de Janeiro, [3. bis 14. Juni 1992] : Schlussbericht der Schweizer Delegation = La Suisse et la conférence de Rio sur l'environnement et le développement / [hrsg. vom Informationsdienst der Direktion für Entwicklungszusammenarbeit und humanitäre Hilfe (DEH)]. - [Bern]: Direktion für Entwicklungszusammenarbeit und humanitäre Hilfe, cop. 1993. - 94 p. - (Schriften der $\mathrm{DEH} ; 3)$.

133.Study on sustainability and social acceptance of the A.P.E. programme among the target population / Susy Greuter. - Bern: DEH; Maputo: Ministère de la santé du Mozambique, 1992.

134.VOEGELE, Kurt ; SOMMER, Martin. - Zusammenarbeit planen : eine Arbeitshilfe für erfahrene Planer und solche, die es werden wollen / Direktion für Entwicklungszusammenarbeit und humanitäre Hilfe, Evaluationsdienst; mit Unterstützung von Josef Imfeid und Malte Lipczinsky. - Bern : DEH, 1992. - 64 p. (Reihe Arbeitshilfen zu Planung, Evaluation, Monitoring und Umsetzung (PEMU)).

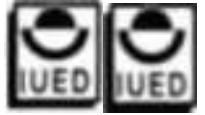

